\title{
Diabetes medications with cardiovascular protection as we enter a new decade: can SGLT2 inhibitors, long-acting GLP-1 receptor agonists, pioglitazone and metformin complement each other to save lives?
}

\author{
ROBERT EJ RYDER ${ }^{1}$, RALPH A DEFRONZO²
}

Key words: type 2 diabetes, cardiovascular outcome studies, SGLT2 inhibitors, GLP-1 receptor agonists, pioglitazone, REWIND, DAPA-HF, PIONEER 6

As we enter a new decade it is timely to reflect on the many advances in pharmacological therapies for type 2 diabetes that occurred with respect to cardiovascular protection during the last 5 years. Every year since the presentation of the EMPA-REG OUTCOME study results in 2015 has seen data from new studies that have increased our understanding, and 2019 was no exception. On 9 June 2019 during the 79th Scientific Sessions of the American Diabetes Association in San Francisco, USA, the results of the REWIND (Researching CV Events with a Weekly INcretin in Diabetes) study were presented and simultaneously published in two Lancet papers. ${ }^{1-3}$ Two days later, at the same event, on 11 June 2019, the results of the PIONEER 6 study were presented and subsequently published in the New England Journal of Medicine. ${ }^{4,5}$ Then, on 19 September 2019 during the European Association for the Study of Diabetes Congress in Barcelona, Spain, the results of the DAPA-HF (Dapagliflozin And Prevention of Adverse-outcomes in Heart Failure) study were presented and published in the New England Journal of Medicine.6,7 Each study has provided new information demonstrating the cardioprotective benefits of long-acting glucagon-like peptide-1 receptor agonists (GLP-1RA) and sodium glucose transporter 2 (SGLT2) inhibitor classes of antidiabetic medications.

In previous editorials we proposed that SGLT2 inhibitors, longacting GLP-1RAs, pioglitazone and metformin in combination could complement each other to prevent cardiovascular events and save lives in patients with type 2 diabetes at high cardiovascular risk..$^{8-12}$ We came to this conclusion because of the accumulated evidence

City Hospital, Birmingham, UK

University of Texas Health Science Center, San Antonio, Texas, USA

Address for correspondence: Dr Bob Ryder

Diabetes and Endocrine Unit, City Hospital, Dudley Road, Birmingham B18 7QH, UK

E-mail: bob.ryder@nhs.net

Br J Diabetes 2020;20:5-8

https://doi.org/10.15277/bjd.2020.250 from multiple studies suggesting that pioglitazone exerts cardiovascular benefit by slowing down, or even reversing, the atherosclerotic process, $^{8-10,12-15}$ whereas SGLT2 inhibitors seem to exert their cardiovascular benefits by improving cardiac haemodynamics and reducing heart failure, ${ }^{8-10,12}$ and possibly by switching myocardial fuel metabolism to ketones. ${ }^{16}$ By contrast, GLP-1RAs appear to exert their cardiovascular benefit by mechanisms different from those of both pioglitazone and SGLT2 inhibitors. ${ }^{12}$ We noted emerging evidence that SGLT2 inhibitors might mitigate the fluid retention associated with pioglitazone, raising the possibility that pioglitazone and SGLT2 inhibitors might complement each other, not only in reducing cardiovascular risk but also in reducing side effects related to fluid retention. ${ }^{17}$ We pointed to the evidence that the early use of triple therapy combination of metformin, pioglitazone and a GLP-1 RA achieved lower $\mathrm{HbA}_{1 \mathrm{c}}$, weight loss and much less hypoglycaemia compared with the traditional approach of sequential escalation through metformin, sulfonylurea and insulin, which was associated with significant weight gain. ${ }^{18}$

REWIND was a randomised controlled trial of the long-acting GLP-1RA dulaglutide versus placebo in 9,901 people with type 2 diabetes. ${ }^{1-3}$ Unlike the previous cardiovascular outcome trials (CVOTs) with GLP-1RAs where the majority of participants had established cardiovascular disease (73-100\%), in REWIND the majority of patients $(68.5 \%)$ only had cardiovascular risk factors. ${ }^{1-3}$ Because of the lower risk of the patients, a much longer trial (median 5.4 years) was required to achieve sufficient cardiovascular endpoints than in the previous CVOTs with GLP-1RA (median 1.6-3.6 years). ${ }^{1-3}$ There was a $12 \%$ reduction in 3-point Major Adverse Cardiovascular Events (MACE: cardiovascular death, non-fatal myocardial infarction and non-fatal stroke) ( $\mathrm{HR}=0.88,95 \% \mathrm{Cl} 0.79$ to 0.99$))^{1,2}$ Figure 1 shows side by side the results of the seven CVOTs (including PIONEER 6 see below) with GLP-1RAs and is in keeping with a class effect for this group of agents. As pointed out previously, ${ }^{11,12}$ the cardiovascular benefit of the GLP-1 RAs is confined to long-acting agents, with no benefit from short-acting lixisenatide (Figure 1). The novel finding from REWIND is that the cardiovascular benefit of dulaglutide was the same whether or not the patients had prior established cardiovascular disease.1,2 It was also noteworthy that similar benefits occurred in men and women and in those with higher and lower body mass index, higher and lower $\mathrm{HbA}_{1 \mathrm{c}}$ and regardless of duration of diabetes. ${ }^{1,2}$ 
Figure 1. MACE results from the seven cardiovascular outcome trials with GLP-1RAs shown side by side with result of meta-analysis also shown. The names of the studies and the agents studied are shown. For more information on these studies see refs $1,2,4,5,9,11,12$

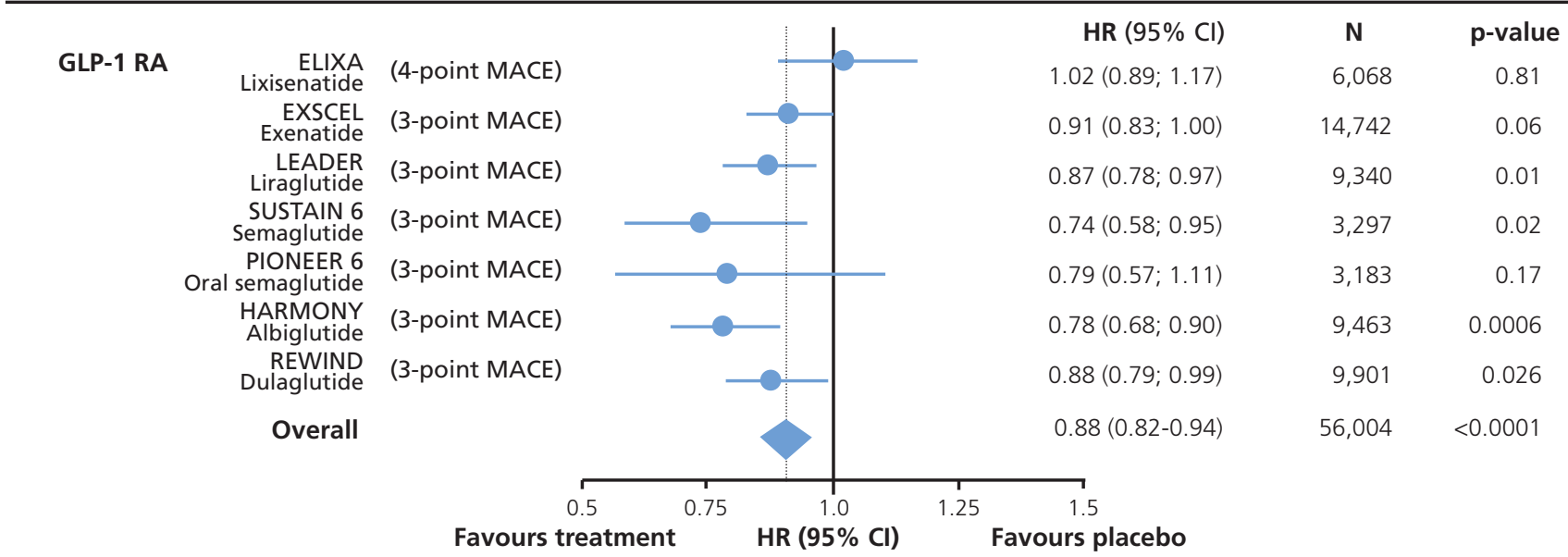

MACE, major adverse cardiovascular event; HR, hazard ratio; Cl, confidence interval. Adapted from references 4 and 23.

Figure 2. Cumulative incidence of all-cause death in the oral semaglutide group versus placebo group in the PIONEER 6 study

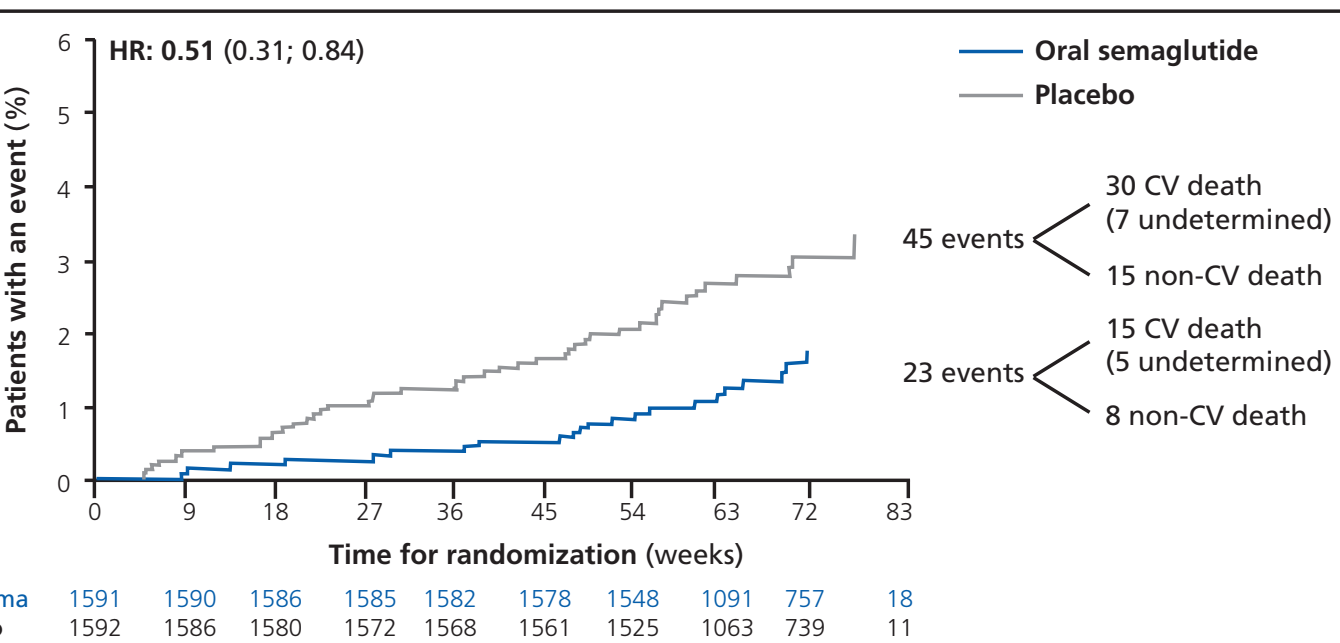

Hazard ratios [HR $(95 \% \mathrm{Cl})]$ based on Cox regression analysis. Adapted from reference 4

PIONEER 6 was a randomised controlled trial of the oral GLP-1RA semaglutide against placebo in 3,183 patients. ${ }^{4,5}$ It was a relatively small trial as the primary objective of the trial was to confirm that treatment with oral semaglutide does not result in an unacceptable increase in cardiovascular risk compared with placebo in patients with a high risk of cardiovascular events. ${ }^{4,5}$ In line with previous CVOTs, PIONEER 6 assessed as its primary outcome 3-point MACE. There was a $21 \%$ reduction in 3-point MACE (HR=0.79, 95\% CI 0.57 to 1.11), which achieved the primary objective by confirming non-inferiority for oral semaglutide versus placebo; however, the reduction in 3-point MACE did not achieve statistical significance. Although the number of events was small and the duration of follow-up short, patients randomised to oral semaglutide experienced a $\sim 50 \%$ reduction in cardiovascular death $(\mathrm{HR}=0.49,95 \% \mathrm{Cl} 0.27$ to 0.92$)$ and all-cause mortality ( $\mathrm{HR}=0.51,95 \% \mathrm{Cl} 0.31$ to 0.84 ) (Figure 2).4,5
DAPA-HF involved 4,744 patients with New York Heart Association class II, III or IV heart failure and an ejection fraction $\leq 40 \%$ who were randomised to dapagliflozin $10 \mathrm{mg}$ or placebo.6,7 The noteworthy feature of this study was that over half of the patients $(58 \%)$ did not have diabetes. The primary endpoint was time to first occurrence of any of the components of the composite worsening heart failure (hospitalisation or an urgent visit resulting in intravenous therapy for heart failure) or cardiovascular death. The outcome was a $26 \%$ reduction ( $\mathrm{HR}=0.74,95 \% \mathrm{Cl} 0.65$ to 0.85$)$ in this endpoint. The individual endpoints of worsening heart failure event and cardiovascular death were both significantly reduced. The patients also experienced significant symptomatic improvement according to the Kansas City Cardiomyopathy Questionnaire. The striking finding from this study was that the improvements were similar whether the patient did ( $\mathrm{HR}=0.75,95 \% \mathrm{Cl} 0.63$ to 0.90$)$ or 
Figure 3. Primary endpoint of worsening heart failure or cardiovascular death in all patients and in those with and without diabetes in the DAPA-HF study

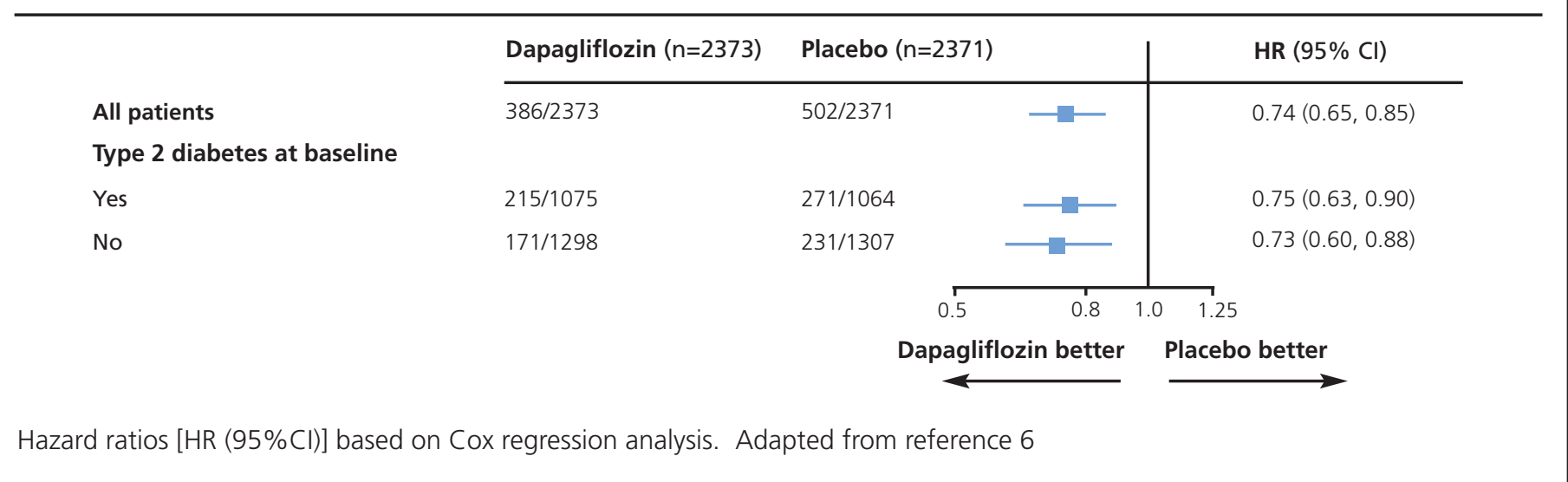

did not ( $\mathrm{HR}=0.73,95 \% \mathrm{Cl} 0.60$ to 0.88 ) have diabetes (Figure 3). 6.7 As we enter a new decade, we believe that by adding together all the trial data which has now accumulated with regard to glycaemic medications with cardiovascular benefit, we can conclude the following:

- Metformin remains difficult to displace as the first-line agent with cardiovascular benefit. Two points in particular are noteworthy: (1) that assessment over many years was required to demonstrate the effect; ${ }^{8}$ and (2) whilst controversial, ${ }^{19}$ it can be argued that the relatively small number of patients in the trial required to demonstrate the outcome is testament to the strength of the effect. ${ }^{20}$ The recent ESC/EASD guidelines ${ }^{21}$ accept the beneficial effect of metformin in primary prevention in newly diagnosed type 2 diabetes without cardiovascular disease (the UKPDS cohort), and the majority of patients in the recent CVOTs received metformin before or concurrently with the newer drugs under test. The relative benefits of metformin compared with the other classes of diabetes medications with cardiovascular benefit are unlikely ever to be established in view of the size, length and cost of any trial that would be required, especially for a drug that is generic.

- Pioglitazone improves cardiovascular outcomes by slowing down or even reversing the atherosclerotic process, independent of its glucose-lowering action..$^{8-10,12-15}$ This is not a class effect for thiazolidinediones and does not apply to rosiglitazone. ${ }^{8}$ The ADAVEASD consensus statement ${ }^{22}$ accepts that pioglitazone has been shown to reduce cardiovascular endpoints, but "without conclusive evidence for benefit". We counter that the only piece of evidence that at first sight raises a question mark over the cardiovascular benefit of pioglitazone is the primary composite endpoint in the PROactive randomised controlled trial, and we have argued that this endpoint is unreliable. ${ }^{13}$ Pioglitazone is contraindicated in patients with heart failure and thus appropriate care needs to be exercised in its use, but in this context it is of interest that SGLT2 inhibitors may mitigate the fluid retention associated with pioglitazone. ${ }^{17}$

- SGLT2 inhibitors as a class seem to exert cardiovascular benefit by improving cardiac haemodynamics and reducing heart failure, ${ }^{8-10,12}$ and possibly by shifting myocardial substrate utilisation. ${ }^{16}$
- SGLT2 inhibitors and pioglitazone in combination may improve cardiovascular outcomes even more by working synergistically to produce benefit in different ways..$^{8-10,12,13}$ As SGLT2 inhibitors seem to mitigate the fluid retention associated with pioglitazone, this further increases their possible complementary actions. ${ }^{17}$

- With regard to dapagliflozin, it is now established that the cardiac benefits are the same for both people with and without diabetes. ${ }^{6,7}$ Given the similarity in cardiac benefit between all the SGLT2 inhibitors, this is likely to be a class effect, but this remains to be established for the other medications.

- There also seems to be a class effect for long-acting GLP-1RAs with regard to improving cardiovascular outcomes, and it seems likely that this class of agents confers benefits by mechanisms that are different from both pioglitazone and SGLT2 inhibitors. ${ }^{12}$

- The cardiovascular benefit has been established across the longacting GLP-1RA class with regard to established cardiovascular disease, but it now seems that, with regard to dulaglutide, the benefit extends to a similar degree to patients who only have cardiovascular risk factors. ${ }^{1,2}$ It is likely that this will also be a class effect for all long-acting GLP-1RAs.

- The cardiovascular benefits of SGLT2 inhibitors can be observed within 1-2 months whereas 1.5-2 years are required to see the cardiovascular benefits of GLP-1 RAs, consistent with the different mechanisms of action of these two classes on the cardiovascular system.

- There is sufficient evidence to suggest that, in an ideal world, trials should be undertaken with combinations of pioglitazone, SGLT2 inhibitors and long-acting GLP-1RAs to establish whether dual or triple therapy does, in fact, confer additional benefit over monotherapy in terms of reducing cardiovascular outcomes and saving lives. It is accepted that the considerable cost of such trials makes them unlikely to be funded using existing systems of funding and agents already on the market. It may be that trials of future agents in these classes might consider containing arms with patients on combinations of agents. It may also be that studies of combinations used in mechanistic studies assessing markers of heart failure or atheromatous cardiovascular disease (as has been done for pioglitazone ${ }^{14,15}$ ) would increase our understanding. 


\section{Key messages}

- The results from the REWIND study with dulaglutide show cardiovascular benefits of this long-acting GLP-1 receptor agonist are the same in patients who only have cardiovascular risk factors as in patients with established cardiovascular disease

- The results of the DAPA-HF trial with dapagliflozin show that the benefits of this SGLT2 inhibitor on heart failure and cardiovascular death are similar for patients both with and without diabetes

- The findings from the REWIND and DAPA-HF trials are likely to reflect class effects for long-acting GLP-1 receptor agonists and SGLT2 inhibitors, respectively

- The results of the PIONEER 6 trial with oral semaglutide demonstrate the cardiovascular safety of this agent. Furthermore, even though the trial was small and of short duration, patients randomised to oral semaglutide experienced a $50 \%$ reduction in cardiovascular death and all-cause mortality

- As we enter a new decade, the accumulated evidence suggests that SGLT2 inhibitors, long-acting GLP-1 receptor agonists, pioglitazone and metformin in combination are likely to complement each other to prevent cardiovascular events and save lives in patients with type 2 diabetes at high cardiovascular risk

Conflict of interest REJR has received speaker fees, and/or consultancy fees and/or educational sponsorships from AstraZeneca, BioQuest, GI Dynamics, Janssen and Novo Nordisk. RAD: Advisory Board: AstraZeneca, Novo Nordisk, Janssen, Boehringer-Ingelheim, Intarcia, Poxel - Honorarium. Research Support: Boehringer-Ingelheim, AstraZeneca, Janssen, Merck - Research Grant - (Investigator). Speaker's Bureau: Novo-Nordisk, AstraZeneca - Honorarium (Speaker)

\section{Funding None}

\section{References}

1. Once Weekly Dulaglutide and Major Cardiovascular Events-Results of the REWIND Trial (Researching CV Events with a Weekly INcretin in Diabetes). Presented on 9 June 2019 during the 79th Scientific Sessions of the American Diabetes Association in San Francisco, USA. Slides available at http://www.phri.ca/REWIND/ (accessed 12 February 2020).

2. Gerstein HC, Colhoun HM, Dagenais GR, et al. Dulaglutide and cardiovascular outcomes in type 2 diabetes (REWIND): a double-blind, randomised placebo-controlled trial. Lancet 2019;394(10193):121-30.

https://doi.org/10.1016/S0140-6736(19)31149-3

3. Gerstein HC, Colhoun HM, Dagenais GR, et al. Dulaglutide and renal outcomes in type 2 diabetes: an exploratory analysis of the REWIND randomised, placebo-controlled trial. Lancet 2019;394(10193):131-8. https://doi.org/10.1016/S0140-6736(19)31150-X

4. Oral Semaglutide-The PIONEER Program Trials. Presented on 11 June 2019 during the 79th Scientific Sessions of the American Diabetes Association in San Francisco, USA. Slides available at https://tracs.unc.edu/PIONEER (accessed 12 February 2020).

5. Husain M, Birkenfeld AL, Donsmark $M$, et al. Oral semaglutide and cardiovascular outcomes in patients with type 2 diabetes. N Engl J Med 2019; 381(9):841-51. https://doi.org/10.1056/NEJMoa1901118

6. EASD VIRTUAL MEETING. Webcast: Dapagliflozin And Prevention of Adverse-outcomes in Heart Failure (DAPA-HF). https://www.easd.org/ virtualmeeting/home.html\#! contentsessions/3167 (accessed 12 February 2020).
7. McMurray JJV, Solomon SD, Inzucchi SE, et al. Dapagliflozin in patients with heart failure and reduced ejection fraction. N Engl J Med 2019;381(21): 1995-2008. https://doi.org/10.1056/NEJMoa1911303

8. Ryder REJ, DeFronzo RA. Diabetes medications with cardiovascular protection in the wake of EMPA-REG OUTCOMETM: the optimal combination may be metformin, pioglitazone and empagliflozin. Br J Diabetes Vasc Dis 2015; 15:151-4. http://dx.doi.org/10.15277/bjdvd.2015.045

9. Ryder REJ, DeFronzo RA. Diabetes medications with cardiovascular protection - what now after LEADER@? Could metformin, pioglitazone, empagliflozin and liraglutide complement each other to save lives? $\mathrm{Br} J$ Diabetes 2016:16:103-6. http://dx.doi.org/10.15277/bjd.2016.096

10. Ryder REJ, DeFronzo RA. What now on the CANVAS of diabetes medications with cardiovascular protection? Could metformin, pioglitazone, SGLT2 inhibitors and liraglutide complement each other to save lives? Br J Diabetes 2017;17:89-92. http://dx. doi.org/10.15277/bjd.2017.036

11. Ryder REJ, DeFronzo RA. Diabetes medications with cardiovascular protection in the wake of EXSCEL - is there a class effect for long-acting GLP-1 receptor agonists? Br J Diabetes 2017; 17:131-3. https://doi.org/10.15277/bjd.2017.147

12. Ryder REJ, DeFronzo RA. Diabetes medications with cardiovascular protection after HARMONY Outcomes and DECLARE-TIMI 58: could metformin, pioglitazone, SGLT2 inhibitors and long-acting GLP-1 receptor agonists complement each other to save lives by different mechanisms? Br J Diabetes 2019;19:1-5. https://doi.org/10.15277/bjd.2019.207

13. Ryder REJ. Pioglitazone has a dubious bladder cancer risk but an undoubted cardiovascular benefit. Diabet Med 2015;32:305-13. http://dx.doi.org/10.1111/dme.12627

14. Mazzone T, Meyer PM, Feinstein SB, et al. Effect of pioglitazone compared with glimepiride on carotid intima-media thickness in type 2 diabetes: a randomized trial. JAMA 2006;296:2572-81. http://dx.doi.org/10.1001/jama.296.21.joc60158

15. Nissen SE, Nicholls SJ, Wolski K, et al. Comparison of pioglitazone vs glimepiride on progression of coronary atherosclerosis in patients with type 2 diabetes: the PERISCOPE randomized controlled trial. JAMA 2008; 299:1561-73. http://dx.doi.org/10.1001/jama.299.13.1561

16. Ferrannini E, Mark M, Mayoux E. CV Protection in the EMPA-REG OUTCOME trial: a "thrifty substrate" hypothesis. Diabetes Care 2016;39:110814. http://dx.doi.org/10.2337/dc16-0330

17. Gautam A, Agrawal PK, Prakash P, Hazra DK. Pioglitazone associated pedal edema resolved by adding sodium glucose co-transporter 2 inhibitor. ADA 76th Scientific Sessions 2016. Late Breaking Poster Session, Poster 140-LB. Abstract 140-LB available at https://diabetes.diabetesjournals.org/content/ diabetes/suppl/2016/06/20/65.Supplement_1.DC1/2016_ADA_LB_Abstracts_HiRes_FINAL_5_11_16.pdf (accessed 12 February 2020).

18. Abdul-Ghani MA, Puckett C, Triplitt $C$, et al. Initial combination therapy with metformin, pioglitazone and exenatide is more effective than sequential add-on therapy in subjects with new-onset diabetes. Results from the Efficacy and Durability of Initial Combination Therapy for Type 2 Diabetes (EDICT): a randomized trial. Diabetes Obes Metab 2015;17:268-75. http://dx.doi.org/10.1111/dom.12417

19. Boussageon R, Gueyffier F, Cornu C. Metformin as firstline treatment for type 2 diabetes: are we sure? BMJ 2016;352:h6748.

https://doi.org/10.1136/bmj.h6748

20. Ryder RE. British Medical Journal Rapid Response to: Metformin as. firstline treatment for type 2 diabetes: are we sure? Available at: https://www.bmj.com/content/352/bmj.h6748/rr-2 (accessed 12 February 2020).

21. The Task Force for diabetes, pre-diabetes, and cardiovascular diseases of the European Society of Cardiology (ESC) and the European Association for the Study of Diabetes (EASD). 2019 ESC Guidelines on diabetes, pre-diabetes, and cardiovascular diseases developed in collaboration with the EASD. Eur Heart J 2020;41:255-323. https://doi.org/10.1093/eurheartj/ehz486

22. Davies MJ, D'Alessio DA, Fradkin J, et al. Management of hyperglycaemia in type 2 diabetes, 2018. A consensus report by the American Diabetes Association (ADA) and the European Association for the Study of Diabetes (EASD). Diabetologia 2018;61(12):2461-98. https://doi.org/10.1007/s00125-018-4729-5.18

23. Kristensen SL, Rørth R, Jhund PS, et al. Cardiovascular, mortality, and kidney outcomes with GLP-1 receptor agonists in patients with type 2 diabetes: a systematic review and meta-analysis of cardiovascular outcome trials. Lancet Diabetes Endocrino/ 2019;7(10):776-85. https://doi.org/10.1016/S2213-8587(19)30249-9 